\title{
DESIGN DIFFERENCES BETWEEN ROBOTIC MANIPULATORS AND CONSTRUCTION EQUIPMENT
}

\author{
Gerry B. Andeen, Staff Scientist \\ SRI International \\ 333 Ravenswood Avenue \\ Menlo Park, California 94025
}

\begin{abstract}
Robotic manipulators and construction equipment come from different traditions that have different objectives. The differences impede use of robotics in construction and use of construction technology in robotics. Robots come from the machine tool tradition where the objective is to hold the position of a tool independent of the forces on it, allowing repetitive production of uniform parts despite differences in stock. The objective of construction equipment is to augment human strength, e.g., to move heavy loads over uneven surfaces. Robotic manipulators have bulky, rigid limbs, the result of designing to put natural mode frequencies above the control system rolloff, so that the control system will not respond to the vibration. The typical maximum payload-to-weight ratio of a robotic manipulator is only about 1:20-far too low for a crane. Construction equipment is designed to be rugged rather than rigid; thus, some bending of a boom or flexing of an outrigger is acceptable. An understanding of the fundamental differences between machine tools and construction equipment can show where improvement would benefit both technologies.
\end{abstract}

\section{INTRODUCTION}

A question often asked, and one that seems to be the underlying question of this conference, is why robotic manipulators and their related technologies have not made more of an impact in construction. One reason may be the uncertainties of the construction environment: today's robots do not have sufficient sensors and brains to maneuver through a construction site and find a poorly specified location. There is an implication, however, that if we wait, the necessary technologies will be developed, perhaps from Artificial Intelligence, so that robotics can be fully applied to construction.

This paper proposes a different answer to the question, with very different implications: robotic manipulators developed to do very different kinds of tasks than are done in construction. An analysis of the differences between typical robotic tasks and construction tasks implies that wider use of robots in construction is not just a matter of waiting until more advanced robotic techniques are developed. Instead, we must recognize the differences between the tasks and make design and control accommodations that are specifically 
for construction tasks. The analysis also suggests that the design of construction equipment can contribute to the improvement of robotics.

\section{THE MACHINE TOOL HERITAGE}

The "machine tool" is a special category of equipment that played a major historical role because it made possible interchangeable parts, which made mass-production possible. Today, machine tools (the lathe, drill press, milling machine) and their modern numericalcontrolled and computer-numerical-controlled multifunction descendants remain essential to our way of life.

The main characteristic of the machine tool is rigidity, the ability to hold position independent of forces. The machine tool makes the same part regardless of whether a large chip or a small chip needs to be removed. Typically the chain or connection from the part (through ground) to the tool is rigid. For example, the part is held tightly in the jaws of a lathe, and those jaws are connected to the frame or bed of the lathe, on which rides the tool holder, holding the tool that will cut excess metal from the part.

The relationship between the machine tool and most currently available robotic manipulators is relatively easy to trace as an extension of numerical control methods. In fact, many of the companies that make robotic manipulators also continue to manufacture classical machine tools. Not surprisingly, the two design methods are similar: the emphasis remains on positional accuracy even though typical robot configurations make that difficult. The control systems used initially were versions of position-control mechanisms on automated machine tools.

The success of the machine tool, the importance of such tools in our society, and the origin of robotics in machine tools have together had a strong influence on the way robotic engineers think. In many cases, the implicit patterns of conceptualization have been limiting, i.e., manipulator ideas do not translate well to the construction environment. Design approaches based on the machine tool school of thought [1] lead naturally to bulky robots that have poor payload-to-weight performance. Infusion of methods from other traditions, i.e., from construction equipment, would enable engineers to design better manipulatorsextend their applications and improve their performance.

\section{MANIPULATORS AND FORCE-CONTROLLED TASKS}

Robotic manipulators have accomplished tasks outside the machine tool tradition using special end-effectors that have built-in flexibility for measuring forces and for deflecting in appropriate ways when contact is made with an object. Examples of tasks accomplished with compliant end-effectors are insertion and grinding, both of which illustrate the differences between position-controlled tasks and force-controlled tasks.

Assembly often requires insertion of a pin or a bolt - the peg-in-the-hole problemwhere the positioning tolerance is not possible with the equipment available. For example, a manipulator with a typical 50-mil accuracy could not locate a one-inch-diameter shaft with sufficient precision to put it into a 1.010-inch-diameter hole (considered a "loose-running fit"). The solution has been to use force feedback-knowledge of the pin-object relationship derived from the interaction forces. Fine craftsmen such as watchmakers also function 
in this way as they assemble parts whose positioning tolerances are much finer than the positioning accuracy of the hand.

Although some grinding demands positional accuracy and a machine tool approach, often the purpose of grinding is only to achieve a smooth surface. The exact position is not important, just that the surface is smoothed by the operation, such as an automobile fender ground down before painting or flashing ground away from a casting. In these cases, position reference derived through forces is fundamental to achieving results, whether done by machine or by man.

\section{DIFFERENCES BETWEEN CONSTRUCTION EQUIPMENT AND MACHINE TOOLS}

At first glance, it would appear that construction equipment does indeed do positioning and is therefore similar in function to a machine tool. A grader levels a field, a backhoe digs a trench along a specified path, and a crane positions a beam according to the plans for a building. The difference has to do with the method of referencing positions, i.e., whether or not a rigid chain is established between the work piece and the tool, and especially with the role of forces in achieving the goal.

Most construction tasks are not tasks where a machine tool approach is possible. It is not possible to have a rigid linkage from the object through the base to the tool, even if it were desired. Three construction examples will illustrate the point:

- Grader - A machine tool approach to grading would be to build a long, rigid beam across the field or along the road to be graded. The leveling would be accomplished by maintaining a constant reference with the rigid beam through a stiff rider. The road grader and scraper uses something of a machine tool approach by having a very long wheelbase, taking the average of several references to the ground to position the blade. The result of several averaging passes is a smoothing. One of the early successes of so called robotic techniques was the laserguided bulldozer blade. Guidance on when to dig deeper and harder relative to the frame came from laser leveling instruments.

- Backhoe-Backhoe operators continually make adjustments according to the hardness of the ground as well as the dimensions of the hole they want to dig. They get force information from the tipping of the platform, and they can "feel" a pipe, cable, or rock and take corrective action. The information they get through the seat of the pants, and also visually, tells them how hard a bite to take. The function of the machine is more to amplify the operator's physical force than it is to follow a well-defined path with each bite. The backhoe is an ideal place to install a forceoriented control system that will augment performance by being more intuitive for the operator; such a system would point the way toward more automatic controls.

- Crane-The crane lifts a beam into position, but close observation makes it clear that the real positioning is done by the ironworker with his drift pin. If the beam is to be placed horizontally, for example, the beam is held by the crane near the middle. This allows the end of the beam to be handled in three directions to position the first end for bolting to an upright. Ironworkers can move the end of the 
beam up and down, changing the orientation of the beam relative to the horizontal. They can move the beam axially, a swinging motion of the cable. Finally, they can move the beam horizontally, normal to its axis, a twisting motion of the cable over which the crane operator has no control. The real role of the crane is not to position the beam, but to unload the weight of the beam so that the ironworker can deal with the positioning.

Although each case is different, the distinction from a machine tool remains quite clear for all. A final differnce is that construction machines are designed for strength and fatigue considerations rather than to meet stiffness standards. In spite of their rugged appearance, pieces of construction equipment are usually expected to deflect during use and the attention to stress and fatigue indicates that deflection is not the primary concern.

\section{ROLE OF FORCE AND COMPLIANCE}

The role of force needs to be included in expanding the role of manipulators beyond machine tools. In construction equipment, the role of force needs to be explicitly recognized and included in control systems.

More particularly, the measurement and application of force implies compliance. Thus it becomes necessary to think in terms of compliance as well as position. We think of the role of the robot as going beyond position control, with its 6 degrees of freedom $(\mathrm{x}, \mathrm{y}, \mathrm{z}, \phi, \theta, \psi)$, to include position and compliance control at the end-point (described by a 6 by 6 symmetric matrix having 21 independent terms that can be adjusted):

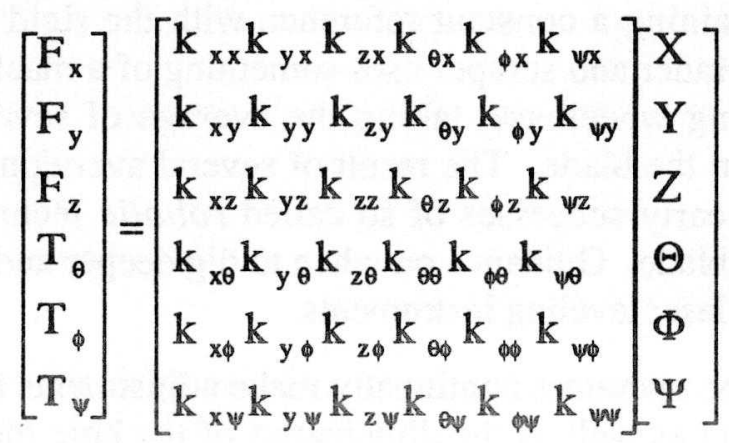

Variable compliance and position affect both structural design and control.

In particular, we advocate the design of structures that are deliberately compliant (a condition usually unavoidable in construction equipment), and that means varying the compliance included in the design. The concept is shown in Figure 1. It is an elbow-type manipulator whose forearm and upper arm are nearly the same length and which a three-roll wrist has been added. This basic two-link manipulator configuration has been shown to have maximum working volume for given limb sizes [2]. The elbow-plus-wrist manipulator consists of a base joint (numbered 1) with a vertical axis of rotation, shoulder (2) and elbow (3) joints whose axes are parallel and horizontal. The joints of the three-rollwrist are 5,6 , and 7 or 4,6 , and 7 . Joints 4 and 5 are kinematically redundant for reasons that will be explained. 


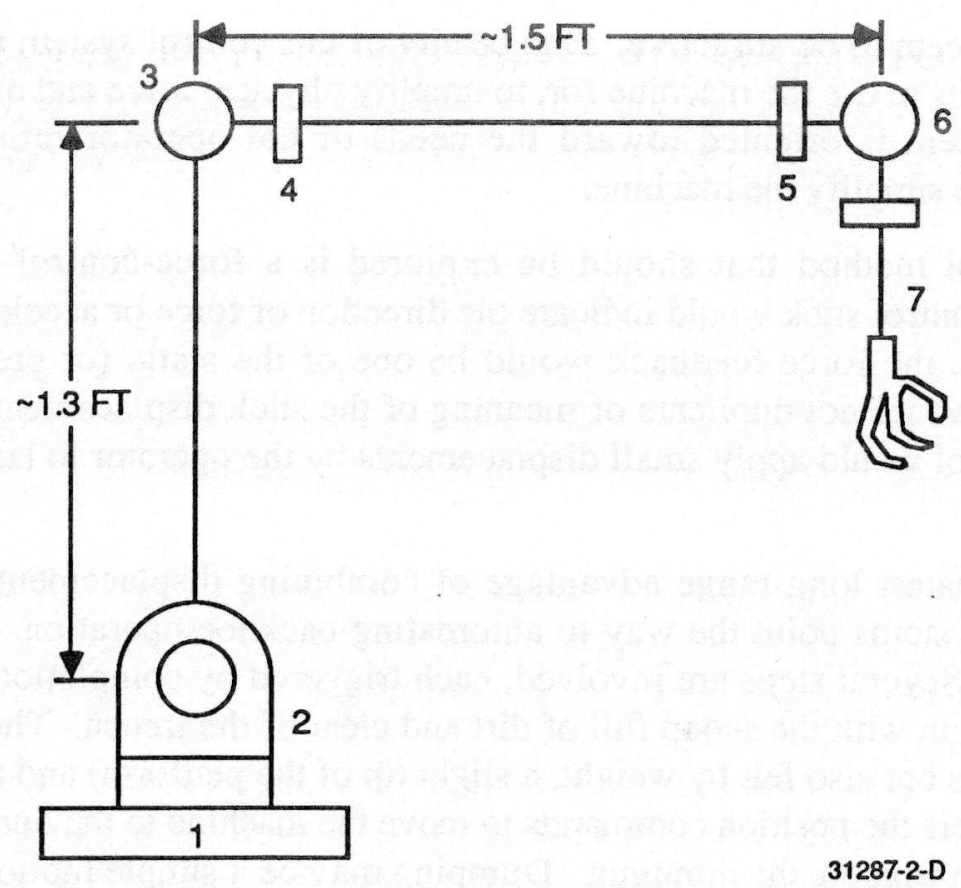

FIGURE 1 SCHEMATIC DIAGRAM OF PROPOSED MANIPULATOR

In our research manipulator, the upper arm is nearly rigid to simplify position-control problems. Compliance resides in the forearm, which has considerably more flexibility in one direction of bending than in the orthogonal direction. This compliant limb is located between Joints 4 and 5 and can be rotated independently of the kinematic configuration. The purpose of the extra joint is to provide variable end point compliance in addition to the position and orientation control provided by the usual six joints.

In control, we advocate increased emphasis on teleoperation and control methods that focus on processes of open-loop force control. The teleoperator concept is crucial, it implies starting with the existing equipment and its method of control rather than assuming a position control perspective.

The backhoe is an ideal place to install a control system more suited to the operator. Today, the typical backhoe uses several hydraulic valves that control the velocity of joints, and the operator must have considerable coordination skills. A simple improvement would be to provide a three-dimensional joystick control where the displacement of the joystick is the velocity command for the end of the backhoe. There would be advantages in operator learning time and ease of use. A more attractive alternative is a position control with force feedback: operators would make the actual motion they want the end of the backhoe to make. At the same time, they would be able to feel the forces of the backhoe against the ground, or rock, or cable, and interpret the dynamics requiredto make the machine move in response to their command. The force feedback need not come through the joystick, 
although that would seem to be attractive. One beauty of this control system is that it fits what the operator wants to use the machine for, to amplify physical force and extend reach. Here,the control system is oriented toward the needs of the operator rather than the manufacturer's need to simplify the machine.

Another control method that should be explored is a force-control stick. The displacement of the control stick would indicate the direction of force or acceleration to be applied. In this case, the force feedback would be one of the static (or preferably the dynamic) forces but would not duplicate of meaning of the stick displacement command. An acceleration control would apply small displacements by the operator to large fields of movement.

Perhaps the greatest long-range advantage of combining displacement with force control is that such systems point the way to automating backhoe operation. Consider a trenching operation. Several steps are involved, each triggered by completion of the previous step. Let us begin with the scoop full of dirt and clear of the trench. That the scoop is full (detected by eye but also felt by weight, a slight tip of the platform) and at a position out of the trench triggers the position commands to move the machine to the dumping point. Reaching this position triggers the dumping. Dumping may be a simple motion, or it may be combined with load measurement so as to spread the dumped load. The empty scoop is the signal to move to a new location relative to the completion of the last scoop, and then downward until the ground is touched. The force signal then begins the important movement that position-controlled robotic manipulators cannot duplicate. The digging motion is a combination of a force applied against the soil together with a motion control normal to the direction of force. Extremes in force, as when the scoop hits rock or a cable, will terminate the motion and trigger a restart with slightly different parameters.

A programming-by-doing approach to automation is the most practical for construction. Conceptually, the operator would begin a repetitive operation-e.g., to dig a trench. The machine would analyze for patterns, identifying first the independent parts of the cycle and then signals for starting and terminating them. The operator would then let the machine take over steps, perhaps first the positioning steps to dump the load or prepare for the next cut. Eventually the machine would be doing the entire repetitive job.

\section{CONCLUSIONS}

Construction offers a different set of tools and tasks than have been considered for robotic manipulators. Typical construction equipment is too flexible to be controlled by current robotic techniques that emphasize position control. Yet construction tools offer design techniques that will have to be included in robotics to make them lighter, and construction tasks point the way to advanced controls that will have to be incorporated to increase the range of tasks manipulators can perform. The control task should begin with improved teleoperator control of construction equipment.

The key to progress is recognizing the fundamental differences between current robotic manipulators and construction equipment and proceeding to cross fertilize the technologies. 


\section{REFERENCES}

[1] Andeen, G.B., R. Kornbluh, 1988: "Design of Compliance in Robotics," Proceedings of the IEEE Intl. Conf. on Robotics and Automation, Philadelphia, PA, Vol 1, pp. 276-281.

[2] Paden, B.E., 1986: "Kinematics and Control of Robot Manipulators," Memorandum No. UCB/ERL M86/5, Electronics Research Laboratory, U. California, Berkeley, CA (January). 